\title{
Okun's Law Across Time and Frequencies
}

\author{
Luís Aguiar-Conraria* Manuel M. F. Martins ${ }^{\dagger} \quad$ Maria Joana Soares ${ }^{\ddagger}$
}

February 28, 2020

\begin{abstract}
We present the first assessment of U.S. Okun's Law across time and frequencies. We use a set of continuous wavelet tools that allows for estimating Okun's coefficient and the lead/lag of output over unemployment at each moment and for each cyclical frequency. We find similar results for the gaps and the first differences specifications at business cycles frequencies, but not at lower frequencies. Okun's coefficient has increased (in absolute value) since the mid1960s, except in 1985-1995, and is not particularly sensitive to recessions. The lead of output varies considerably along time and also at different frequencies. We observe (especially with the gaps specification) that there are at least two cyclical processes relevant for the Okun's relationship. One at the business cycle and another at lower frequencies. Methods that do not take this into account are bounded to mix the information embedded in both cycles.
\end{abstract}

Keywords: Okun's Law; Unemployment; Business Cycles; Wavelet Gain; Time-Frequency Estimation; Continuous Wavelet Transform.

JEL codes: C49, E24, E32.

*NIPE and Department of Economics, University of Minho, E-mail address: lfaguiar@eeg.uminho.pt. Corresponding author.

${ }^{\dagger}$ Cef.up and Faculty of Economics, University of Porto, E-mail address: mmfmartins@fep.up.pt

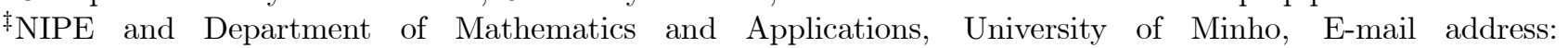
jsoares@math.uminho.pt 


\section{Introduction}

Okun (1962) uncovered a negative short-run relation between cyclical unemployment and cyclical real output, which proved to be such a remarkable stylized fact that came to be known as Okun's Law. His two key econometric specifications, adopted in the subsequent literature, describe a relation either between levels or between the first differences of output and unemployment

$$
\begin{gathered}
u_{t}-u_{t}^{*}=\alpha+\beta\left(y_{t}-y_{t}^{*}\right), \\
\Delta u_{t}=\mu+\gamma\left(\Delta y_{t}\right),
\end{gathered}
$$

in which $u_{t}$ is the unemployment rate in period $t, u_{t}^{*}$ is the natural rate of unemployment, $y_{t}$ is the logarithm of real output, $y_{t}^{*}$ is the logarithm of potential output, and $\Delta$ is the first difference operator.

In Okun's original estimates, a $1 \%$ deviation of real output from potential was associated with about a $0.33 \%$ deviation of unemployment from trend (levels or gaps specification), as a $1 \%$ change in real output was associated with about a $0.33 \%$ change in the unemployment rate (first differences specification). ${ }^{1}$ Okun's view was that each percentage point of deviation of output from trend would be divided into a change of a third of a percentage point in productivity and a change of two thirds of a percentage point in aggregate hours, subdivided into a change of one third of a percentage point in the unemployment rate and roughly equal changes in labor force participation and hours per employee - see Gordon (2010a, 2010b).

Subsequent empirical research on Okun's Law established the rule of thumb that, in the U.S., each $1 \%$ deviation of real output from potential is associated with about $0.5 \%$ deviation of unemployment from the natural rate. ${ }^{2}$ Okun's Law has been omnipresent in macroeconomics textbooks,

\footnotetext{
${ }^{1}$ Okun (1962) obtained similar estimates of the coefficient for the first differences and the gaps specifications, because he assumed a constant natural rate and unemployment and a constant trend growth rate of output.

${ }^{2}$ Some authors derived the Okun's Law from its first principles - the production function - and often failed to confirm either Okun's original coefficient (0.33) or the rule of thumb coefficient omnipresent in textboook and applied analyses (0.5) - see, e.g., Prachowny (1993).
} 
macro models with unemployment, and applied business cycles analyses. Moreover, it has been the focus or a key analytical tool in a vast academic literature, recently earning a renewed interest - see e.g. Ball, Leigh and Loungani (2017), Fernald, Hall, Stock and Watson (2017), Kamber, Morley and Wong (2018), and Arai (2016).

However, the stability of the coefficient of Okun's Law has been challenged by a large literature in two ways: (1) the possibility of nonlinearities (meaning that the coefficient may be different at different phases of the business cycle); (2) time-variations due to possible structural breaks or changing trends. Moreover, some (smaller) literature has challenged the stability of the timing of Okun's Law, raising issues related to its correct lag specification over time.

While Okun's coefficient may have been unstable across time, it is likely that it has also been unstable for different frequencies. Not only there is evidence that cycles longer than those associated with business cycle frequencies display relevant information for macroeconomic analysis (Pancrazi, 2015), but there is also evidence of a lengthening of business cycles (Aguiar-Conraria, Martins and Soares, 2018, and Crowley and Hallett, 2018). If such volatility transfer has been caused by a change in policy preferences, then it very likely has also affected other main macroeconomic variables, such as the unemployment rate, and their relation with output. To test for this possibility, one has to scrutinize Okun's Law at different frequencies, which the literature has not done so far.

Our paper adds to this important literature by using a technique that allows us to simultaneously deal with all these issues, namely, instability over time due to nonlinearities, time-variations, and changing lags specifications, as well as instability over frequencies.

We use continuous wavelet tools, with an approach consisting of a sequential analysis of wavelet coherencies, phase-difference diagrams, and wavelet gains, that provides estimates of the U.S. Okun's Law coefficient simultaneously allowing for variations over time as well as variation in the timing of the relationship and variation across frequencies. More specifically, coherency indicates the strength and significance of the Okun's relationship at each frequency for each moment of time, the gain provides an estimate of Okun's coefficient at each frequency and moment of time, and the phase-difference indicates the sign of the estimate and the lead/lag of output relative to unemployment. 
With this set of tools, this paper uncovers new stylized facts about the U.S. Okun's Law in the past seven decades that would be difficult to detect either in the pure time-domain or in the pure frequency-domain. For completeness, we perform our analyses both for the levels (gaps) version and for the first differences version of the Okun's Law.

The paper is structured as follows. In Section 2, we discuss the literature on Okun's law, clarifying our net contributions to the literature. In Section 3, we briefly describe our methodology. In Section 4 we present the data and perform a preliminary time-frequency analysis of each time series. In Section 5, we present our results of estimation of Okun's Law in the time-frequency domain. Section 6 concludes, presenting in a systematic way the stylized facts uncovered in the paper and comparing these with the current literature on U.S. Okun's Law. 


\section{The Evolution of Okun's Law Since 1962}

The stability of the coefficient of Okun's Law has been challenged by a large literature. The literature has focused on two key aspects, both of which are automatically dealt with our wavelet approach: nonlinearities and time-variations. Nonlinearities are related, for example, with possible asymmetries over the business cycle. Time-variations may occur due to structural breaks or changing trends.

Regarding nonlinearities, many authors found that each percentage point of cyclical output is associated with a larger (in absolute value) reaction of the cyclical unemployment rate in recessions as compared to expansions - e.g., Lee (2000), Owyang and Sekhposyan (2012), Cuaresma (2003), Silvapulle, Moosa and Silvapulle (2004), Holmes and Silverstone (2006), Knotek II (2007), Meyer and Tasci (2012), Valadkhani and Smyth (2015), Berger, Everaert and Vierke (2016), and Grant (2018). In contrast, Fernald, Hall, Stock and Watson (2017) did not find different Okun's coefficients for periods of increase and decrease of the unemployment rate. There are many possible reasons for cyclical unemployment to react more to cyclical output in recessions, such as factor substitution (labor force participation and hours versus capital), productivity changes, changes in the sectorial composition of output, labor mismatch, asymmetric adjustment costs, the bargaining power of insiders versus outsiders, and the sharpness and persistence of employer's pessimism - see, e.g., Silvapulle, Moosa and Silvapulle (2004), Valadkhani and Smyth (2015), and Berger, Everaert and Vierke (2016); moreover, asymmetry in Okun's coefficient over the business cycle tends to be larger in countries with labor market institutions that provide less employment protection, such as the U.S. - see Cazes, Verick and Hussami (2013).

Regarding structural changes, many authors found a structural break in Okun's Law, whereby each percentage point of cyclical output is associated with a larger (in absolute value) reaction of the cyclical unemployment rate in recent decades; most of the literature locates the break in the mid-1980s, i.e., the beginning of the Great Moderation - e.g., Knotek II (2007), Gordon (2010a, 2010b), Meyer and Tasci (2012), and Grant (2018) - but others identify a significant change in Okun's coefficient only in the 2001 recession - e.g., Berger, Everaert and Vierke (2016). In contrast with most papers, some authors, such as Owyang and Sekhposyan (2012) and Valadkhani and 
Smyth (2015), found smaller (in absolute value) Okun's coefficients after the beginning of the Great Moderation.

The literature has identified several possible causes for the increase in the Okun's coefficient in the Great Moderation, focusing on changes in the cyclical behavior of productivity (from procyclical to counter-cyclical, with a longer lead ahead of output) and of hours (to a stronger albeit lagged reaction to output); these are ultimately related to an increase in income inequality, an increased tendency of firms to treat workers (especially low-skilled) as a disposable commodity, an increased flexibility of the labor market (given matching and work through information technologies and the increase in part-time involuntary employment), as well as the increase in the share of services in output - see, e.g., Gordon (2010a, 2010b) and Grant (2018); moreover, Dixon, Lim and Van Ours (2017) found that, controlling for other labor market institutions, the Okun's coefficient is positively associated with the share of temporary workers, which has been increasing in the U.S. and therefore also partly explains an increase in the Okun's coefficient.

Yet, some recent papers did not find evidence of a significant structural change in Okun's coefficient. Daly, Fernald, Jordà and Nechio (2017), although acknowledging that productivity changed from pro-cyclical to counter-cyclical and that firms changed their adjustments to shocks in favor of extensive margins, estimate a roughly stable coefficient before and after 1984. Ball, Leigh and Loungani (2017) specified Okun's regressions (both in levels and in first differences) adding two lags of output as explanatory variables, and found that although the estimates of Okun's coefficient are somewhat larger after 1984, the difference has neither statistical nor economic relevance. Their results are consistent with the findings by Galí, Smets and Wouters (2012), who - when many observers were labeling the post Great Recession period as a jobless recovery - found no evidence of change in Okun's coefficient, noting that what happened was that all the recoveries following the 1991:I, 2001:IV and 2009:II troughs had been slow recoveries. ${ }^{3}$

\footnotetext{
${ }^{3}$ Fernald, Hall, Stock and Woston (2017) show that the recovery after 2009:II was unexpectedly slower than the previous three (after the troughs in 1982:IV, 1991:I and 2001:IV) in what regards output but not unemployment. They estimated a constant Okun coefficient throughout 1981:III-2016:II to compute cyclically adjusted output correcting its path from the deepness of the recession and the normal recovery of unemployment - concluding that its shortfall (in the seven years after 2009:II, compared with the seven years after the previous troughs) was of about 1.99 percentage points per year and larger than the shortfall in raw output growth. Ultimately, they show that two thirds of the shortfall is explained by slower trend growth caused by a decline in labor participation force and a slowdown of total factor productivity - trends that were in force before the Great Recession.
} 
The results in Ball, Leigh and Loungani (2017) and in Galì, Smets and Wouters (2012), taken together with the finding in Knotek II (2007) that, after 1990, lagged output dominated contemporary output in explaining the unemployment rate, suggest that the literature has overlooked the role of lags in the specification of Okun's Law. Research has focused on variation in Okun's coefficient largely disregarding the possibility of changes in the timing of the relation between cyclical output and cyclical unemployment. A fortiori, literature assuming purely static Okun regressions with constant coefficients is particularly questionable, even if estimating the Okun's slope - as for example Kamber, Morley and Wong (2018) - and more so if imposing the baseline Okun's coefficient of -0.5 - as for example Arai (2016). In contrast, Fernald, Hall, Stock and Watson (2017) estimate the first differences version of Okun's Law with two leads and lags, which, as will be seen below, is a more robust time series approach to Okun's Law.

Therefore, there is the need to estimate the Okun's Law coefficient with an econometric technique that allows for endogenously selecting the lead or lag relationship between the unemployment rate and real output; this is one of the attributes of our econometric approach in this paper.

So far the literature has been silent regarding frequency issues. However there is not a strong reason to believe the Okun's law to be frequency invariant. Quite the contrary, the evidence of slow recoveries may be related with the important findings of Crowley and Hughes Hallett (2015, and 2018) regarding the length of business cycles, the frequency and amplitude of recessions, and the speed of recoveries and expansions, since the Great Moderation. Crowley and Hughes Hallett established that the Great Moderation has been characterized by a transfer of volatility of output growth from the short-end of business cycles - cycles with up to four years - to very long cycles - with length above 16 years - and maybe even intermediate-frequency business cycles - between four and eight years - and intermediate cycles with eight to 16 years.

Within a canonical New Keynesian model, Crowley and Hughes Hallett (2018) showed that such volatility transfer could have been caused by the increase in the aversion to inflation, relative to output stabilization - featured by increasingly independent and inflation targeting central banks since the mid-1980s, including the FED - with no changes in the parameters governing the structural dynamics of the economy. Their hypothesis finds support in literature that has robustly identified monetary policy changes as key for the Great Moderation - e.g. Coibion and 
Gorodnichenko (2011), who pointed out the enhanced macroeconomic stability arising from the simultaneaous reduction of trend inflation and increase in the reaction of policy to inflation, and Bjørnland, Larsen, and Maih (2018), who have shown that any episodes of heightened macroeconomic volatility have been briefer because of a more credible monetary policy regime responding more to inflation. Such transfer of volatility is an expression of the trade-off known as "design limit" in optimal control theory, formally studied by Brock, Durlauf and Rondina (2008, 2013), whereby feed-back policy rules that reduce variance at some frequencies induce increases in variance at others. A corollary is that if policy preferences were to revert in favor of short-term output stabilization relative to medium-run inflation stabilization and anchoring of inflation expectations, a shift of variance from long cycles to short business cycles would follow. With the current policy preferences, Crowley and Hughes Hallett (2018) predict less frequent and more severe booms and busts, with milder expansions in between.

While the transfer of volatility across frequencies in output growth is a key business cycle change, with very likely impacts on the other main macroeconomic variables and their relation with output, the literature on Okun's Law has not assessed, so far, the relation between cyclical output and cyclical unemployment at different frequencies. Therefore, there is the need to estimate the Okun's Law coefficient with an econometric technique that allows for different estimates at different cyclical frequencies; this is another of the attributes of our econometric approach in this paper. 


\section{Methodology}

The continuous wavelet transform is becoming an increasingly popular tool in econometric analysis. Because of its time-frequency domain approach, it can simultaneously detect patterns that change over time - akin to dynamic conditional correlation (DCC) models and other time varying models - and across frequencies - like spectral analysis. Moreover, given its local nature, wavelet analysis is very suitable to deal with non-stationary data and nonlinear relations. The chapters included in Gallegati and Semmler (2014) provide a review of recent developments.

In this paper, we use the most common wavelets tools - the wavelet coherency, the wavelet phase-difference, and the instantaneous time-lag - and also a recent development that allows us to estimate coefficients akin to regression coefficients in the time-frequency domain - the wavelet gain. Regarding the first set of tools, the reader is referred to Aguiar-Conraria and Soares (2014), who provide a very thorough and technical review, and to Aguiar-Conraria, Magalhães and Soares (2012 and 2013), who provide a more intuitive description of these tools. Regarding the latter concept, the wavelet gain, the reader is referred to Aguiar-Conraria, Soares, and Sousa (2018) and Aguiar-Conraria, Martins, and Soares (2018), who generalize the concept of the wavelet gain and apply it to study carbon markets and monetary policy, respectively.

In what follows, except for the wavelet gain, which, given its novelty, we discuss more carefully, we provide a short overview of these concepts. The knowledgeable reader may skip to next section without loss.

\subsection{The Continuous Wavelet Transform}

In spectral analysis, a time-series is decomposed in a weighted sum of complex exponentials (or, equivalently, into a weighted sum of sines and cosines). In wavelet analysis, the sines and cosines are replaced by a wavelet. A wavelet $\psi(t)$ is a function that oscillates around the $t$-axis (just like sines and cosines), but that looses strength as it moves away from the center, behaving like a small wave. The specific wavelet we use in this paper is the complex-valued function (selected from the Morlet wavelet family) defined by $\psi(t)=\pi^{-\frac{1}{4}} e^{6 i t} e^{-\frac{t^{2}}{2}}$.

Given a time-series $x(t)$, its continuous wavelet transform (CWT), with respect to a given 
wavelet $\psi$, is the function of two variables, $W_{x}(\mathrm{t}, \mathrm{s})$, given by

$$
W_{x}(\mathrm{t}, \mathrm{s})=\frac{1}{\sqrt{|\mathrm{s}|}} \int_{-\infty}^{\infty} x(t) \bar{\psi}\left(\frac{t-\mathrm{t}}{\mathrm{s}}\right) d t
$$

Note that $\mathrm{s}$ is a scaling parameter and $\mathrm{t}$ a translation parameter controlling, respectively, the width and the location along the $t$-axis of the function $\psi\left(\frac{t-\mathrm{t}}{\mathrm{s}}\right)$; for $|\mathrm{s}|>1$, the function becomes larger (hence, corresponding to a function with lower frequency) and for $|s|<1$, it becomes narrower (hence, becoming a function with higher frequency); in the above formula and throughout the paper, an over-bar denotes complex conjugation.

Remark 1 As for the CWT, all the wavelet measures that we are going to introduce are functions of the two variables, $\mathrm{t}$ and $\mathrm{s}$. To simplify the notation, we will describe these quantities for a specific value of the argument, $(\mathrm{t}, \mathrm{s})$, which will be omitted in the formulas.

Similarly to the terminology used in the Fourier case, the (local) wavelet power spectrum is defined as

$$
(W P S)_{x}=W_{x} \overline{W_{x}}=\left|W_{x}\right|^{2}
$$

The wavelet power spectrum gives us a measure of the variance distribution of the time-series in the time-frequency plane. When the average is taken over all times, we obtain the global wavelet power spectrum:

$$
(G W P S)_{x}=\int_{-\infty}^{\infty}\left|W_{x}\right|^{2} d t
$$

which gives us essentially the same information as the Fourier Power spectrum.

If one wants to study the relation between two variables in the time-frequency domain, then one needs to make use of cross wavelet tools. The cross-wavelet transform of two time-series $y(t)$ and $x(t)$, denoted by $W_{y x}$, is defined as $W_{y x}=W_{y} \overline{W_{x}}$, and its absolute value is referred to as the cross-wavelet power. The cross-wavelet power of two time-series depicts the covariance between two time-series at each moment in time and frequency.

Most of the times, in economics, more than in the covariance, we are interested in the correlation between variables. For that, we have the complex wavelet coherency of $y$ and $x$, given by $\varrho_{y x}=$ 
$\frac{S\left(W_{y x}\right)}{\left[S\left(\left|W_{y}\right|^{2}\right) S\left(\left|W_{y}\right|^{2}\right)\right]^{1 / 2}}$, where $S$ denotes a smoothing operator in both time and scale. For simplicity, we will denote by $S_{y x}$ the smoothed cross-wavelet transform of two series $y$ and $x$, i.e., $S_{y x}=S\left(W_{y x}\right)$; we will also use $\sigma_{x}$ to denote the square root of the smoothed wavelet power of series $x$, i.e., $\sigma_{x}=\sqrt{S\left(\left|W_{x}\right|^{2}\right)}=\sqrt{S_{x x}}$, and use similar notation for series $y$. Hence, the formula for the complex coherency can simply be written as:

$$
\varrho_{y x}=\frac{S_{y x}}{\sigma_{y} \sigma_{x}} .
$$

The wavelet coherency, which we will denote by $R_{y x}$, is the absolute value of the complex wavelet coherency, i.e., $R_{y x}=\left|\varrho_{y x}\right|=\frac{\left|S_{y x}\right|}{\sigma_{y} \sigma_{x}}$.

With a complex-valued wavelet, we can compute the phase of the wavelet transform of each series and, by computing their difference, we can obtain information on the possible delays between the oscillations of the two series, as a function of time and frequency. It follows immediately that the wavelet phase-difference between $y$ and $x$, which we will denote by $\phi_{y x}$, can also be computed as the angle of the cross-wavelet transform, $W_{y x}$.

Another slightly different way to define the phase-difference, which has the advantage of allowing a more direct generalization for the multivariate case, makes use of the angle of the complex wavelet coherency, instead of the angle of the cross-wavelet transform:

$$
\phi_{y x}=\arctan \left(\frac{\mathfrak{I}\left(\varrho_{y x}\right)}{\mathfrak{R}\left(\varrho_{y x}\right)}\right) .
$$

A phase-difference of zero indicates that the time-series move together at the specified frequency; if $\phi_{x y} \in\left(0, \frac{\pi}{2}\right)$, then the series move in phase, but the time-series $x$ leads $y$; if $\phi_{x y} \in\left(-\frac{\pi}{2}, 0\right)$, then it is $y$ that is leading. More relevant in this paper is the interpretation of the other quadrants: a phase-difference of $\pi$ or $-\pi$ indicate an anti-phase relation (note that given the circular nature of the angle, it is indifferent to have $\pi$ or $-\pi)$; if $\phi_{x y} \in\left(\frac{\pi}{2}, \pi\right)$, then $y$ is leading. Time-series $x$ is leading if $\phi_{x y} \in\left(-\pi,-\frac{\pi}{2}\right)$.

One can easily convert the phase-difference into the instantaneous time-lag between the 
two time series $y$ and $x$ :

$$
(\Delta T)_{y x}=\frac{\phi_{y x}}{2 \pi f}
$$

where $f$ is the frequency corresponding to the scale s. ${ }^{4}$

\subsection{The wavelet gain}

The wavelet gain is a concept analogous to the Fourier gain. Usually one interprets the Fourier gain as the modulus of the regression coefficient of $y$ on $x$ at a given frequency (see, e.g., Engle 1976). Mandler and Scharnagl (2014) used the same interpretation for the wavelet gain in a given moment in time, at a specific frequency. Aguiar-Conraria, Soares and Sousa (2018) and AguiarConraria, Martins and Soares (2018) generalized the concept to $n$ variables. With this tool at hand, one is finally able to estimate not only time-varying but also frequency varying coefficients. A nice example of the usefulness of this approach is provided by Verona (2020), who assesses the investment-Q sensitivity at different frequencies and its evolution over time, as well as the interaction of the financial cycle with the Q theory.

Naturally, this could also be done with the more traditional tools. For example, one could filter the data using a band-pass filter, focusing the analysis on a particular frequency band, and then estimate a time-varying model for the filtered time-series. Alternatively, one could also rely on the discrete wavelet transform to perform such task. A neat application of the DWT to a subject related to ours, the Phillips curve is provided by Gallegati et al. (2011). With the (partial) wavelet gain, this can be done in one only step, in an almost automatic and very natural way comprising the whole set of frequency ranges.

The complex wavelet gain of $y$ over $x$, denoted by $\mathcal{G}_{y x}$, is defined as $\mathcal{G}_{y x}=\frac{S_{y x}}{S_{x x}}=\varrho_{y x} \frac{\sigma_{y}}{\sigma_{x}}$. We call wavelet gain, and denote by $G_{y x}$, the modulus of the complex wavelet gain, i.e.,

$$
G_{y x}=\frac{\left|S_{y x}\right|}{S_{x x}}=R_{y x} \frac{\sigma_{y}}{\sigma_{x}} .
$$

\footnotetext{
${ }^{4}$ Due to the oscillatory nature of the wavelet $\psi$, it is natural to associate to it a certain frequency and hence, to associate a frequency, depending on the scale parameter s, to each function $\psi\left(\frac{t-t}{\mathrm{~s}}\right)$; in the particular case of the Morlet wavelet here used, we have $f=f(\mathrm{~s}) \approx 1 / \mathrm{s}$. See Aguiar-Conraria and Soares (2014) for details.
} 
Note that one can also use the complex wavelet gain to compute the wavelet phase-difference: $\phi_{y x}=\arctan \left(\frac{\Im\left(\mathcal{G}_{y x}\right)}{\mathfrak{R}\left(\mathcal{G}_{y x}\right)}\right)=\arctan \left(\frac{\Im\left(\varrho_{y x} \frac{\sigma y}{\sigma x}\right)}{\mathfrak{R}\left(\varrho_{y x} \frac{\sigma y}{\sigma_{x}}\right)}\right)=\arctan \left(\frac{\Im\left(\varrho_{y x}\right)}{\mathfrak{R}\left(\varrho_{y x}\right)}\right)$. JOANA CONFIRMA ESTA FRASE QUE AQUI ACRESCENTEI SOBRE A DIFERENÇA DE FASE USANDO O COMPLEX GAIN.

The interpretation of the wavelet gain as a regression coefficient must be done with care. What we do is the wavelet counter-part of what Engle (1976) described as: "The regression coefficient is just the gain if there is no time lag between the independent and dependent variables. If there is a time lag, the gain can be interpreted as the regression coefficient if the series were lagged just the right amount to eliminate any phase shift, and the phase is the angle by which they would have to be shifted". This is an advantage but adds some complexity to the analysis.

The advantage is that our regressions endogenously deal with the variation that may occur, over time and frequencies, in the lag between the variables of interest - automatically adjusting the specifications regarding their time-lags. A change in the time-lag of the relation between cyclical output and cyclical unemployment would imply that a typical regression would become missspecified, and this would contaminate the regression coefficient estimate. This does not happen in our setup.

The additional complexity is that we must complement the analysis of the wavelet gain with that of the phase-difference (or the instantaneous time-lag), to become aware of the time shifts that may occur and correctly interpret the meaning of the coefficient estimated at each time and frequency. 


\section{The Data}

Our data are U.S. quarterly time-series of real output and the civilian unemployment rate for 1948:I-2018:II, provided by the U.S. Bureau of Economic Analysis and the U.S. Bureau of Labor Statistics, respectively, and quarterly estimates of real potential output and the (long-term) natural rate of unemployment, provided by the U.S. Congressional Budget Office (CBO), for 1949:I-2018:II. ${ }^{5}$ We perform our computations using a Matlab toolbox, called ASToolbox v.2018, available at http://sites.google.com/site/ aguiarconraria/joanasoares-wavelets.

We use official data, rather than estimate our own natural rates and gaps (as often done in the literature), for two reasons: first, natural rates estimated by the $\mathrm{CBO}$ are based on a production function approach, which is the closest to the theoretical foundations of Okun's Law; second, any statistical filter (even if hybrid, i.e., combined with macroeconomic equations, as most models of unobservable components) would extract cycles with a limited time-span, necessarily imposed a priori in the case of pure statistical filters (e.g., the Hodrick-Prescott (1997) filter extracts fluctuations with a period between two and 32 quarters), therefore disregarding from the outset cycles that are increasingly likely to be relevant - see, e.g., Canova (2007) for a comparison of methods extracting cyclical information.

Although our preferred specification of Okun's Law relates the gaps of unemployment and output, for completeness and robustness check, we also estimate the first differences version of Okun's Law in the Appendix. ${ }^{6}$ We now briefly describe these data.

In Figure 1 we present a description of the data to be used in the levels (gaps) version of Okun's Law. The output gap is the difference between the natural logarithm of real output and the natural logarithm of real potential output, while the unemployment gap if the difference between the civilian unemployment rate and the long-term natural rate of unemployment, both measured in percentage points.

\footnotetext{
${ }^{5}$ All data have been downloaded from the FRED Economic Database of the Federal Reserve Bank of St. Louis, available at https://fred.stlouisfed.org.

${ }^{6}$ While the gaps version of Okun's Law may be derived from its first principles - a production function - its conversion to the changes specification requires a constant natural rate of unemployment and a constant trend growth rate of output. We follow Ball, Leigh and Loungani (2017) in preferring the gaps version and in estimating also the changes version for robustness and comparison with some literature.
} 

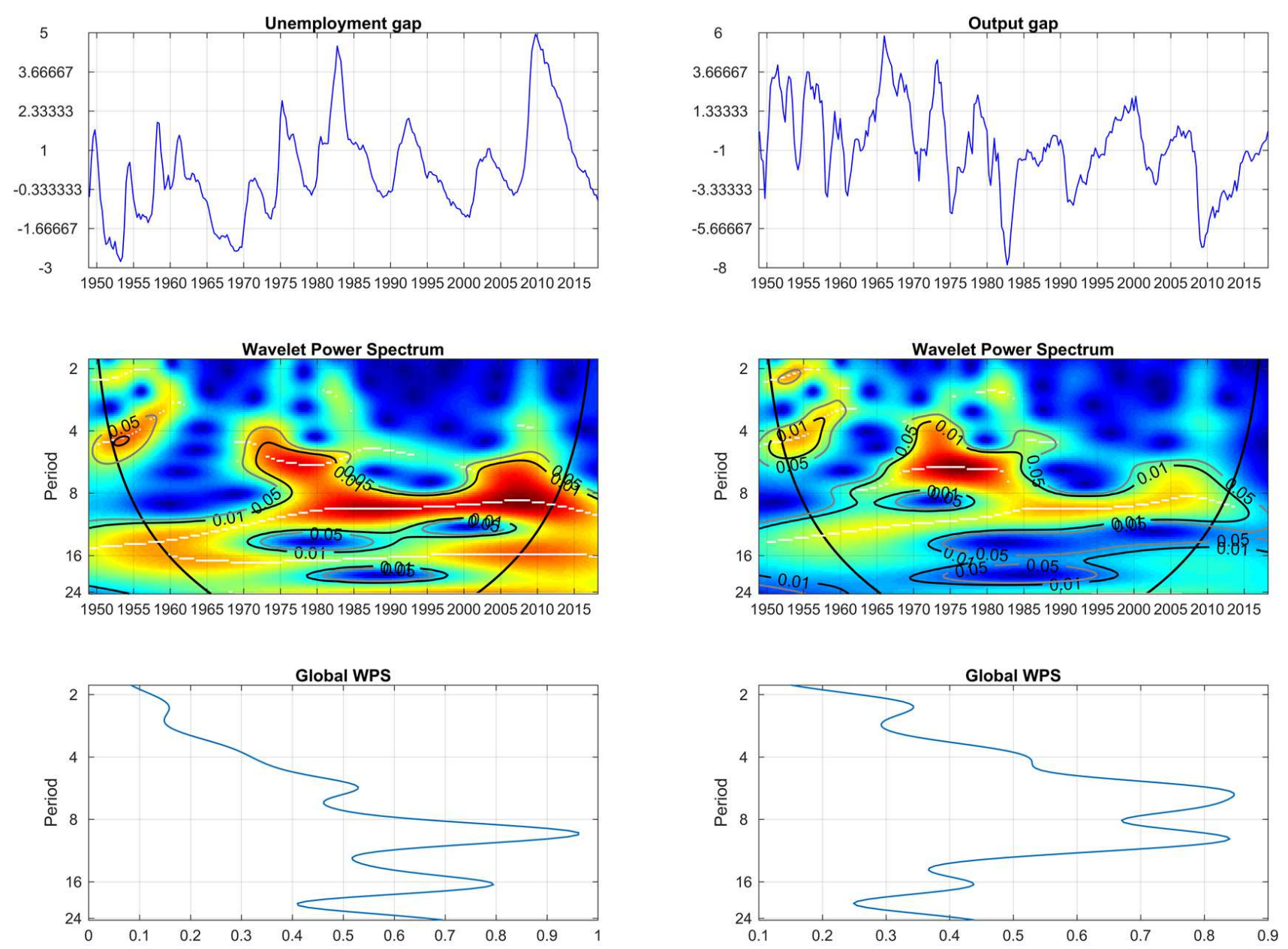

Figure 1. Top: Time-series plots of the unemployment and the output gaps. Bottom: Global power spectrum, showing the variance of each series at each frequency in the overall sample. Middle: Wavelet power spectrum, showing the variance of each series at each time-frequency locus. The color code for power ranges from blue (low power) to red (high power). The white lines show the local maxima of the wavelet power spectrum. The black (grey) contours indicate the 1\% (5\%) significance level. The cone of influence, which indicates the region affected by edge effects, is shown with a parabola-like black line. (for interpretation of the references to color in this legend, the reader is referred to the web version of this article.)

In addition to the standard time-series graphs (top pictures), we present the wavelet-based global power spectra (bottom pictures) - a frequency domain measure, analogous to the Fourier spectrum, which describes the intensity of the variation of the series for each frequency over the whole sample - and the wavelet power spectra (pictures in the middle) - a time-frequency domain measure indicating the variance of the series for each frequency and at each time. A first overall conclusion is that, with the exception of the 1950s and of the first half of the 1970s, the variability of both the output and unemployment gap occurs at frequencies corresponding to periods larger 
than four years. A second overall conclusion is that shorter cycles gradually loose relevance to longer cycles since the oils shocks and until the Great recession, in line with the findings of Crowley and Hallett (2015 and 2018). A third overall conclusion is that, while having many similarities - such as the decrease in volatility at standard business cycles $(4 \sim 8$ years) during the Great Moderation and the increase in volatility at the lower end of such cycles ( $6 \sim 8$ years) ahead and during the Great Recession - the cyclical patterns of the output gap and the unemployment gap do not exactly match when seen with time-frequency domain lenses.

The time series chart of the output gap shows the strong recessions associated with the first oil shock in the mid-1970s and with disinflation in the early 1980s, and then the Great Moderation between 1984 and 2007, and the Great Recession starting at the end of 2007. The wavelet global power spectrum identifies two dominant cycles in the output gap throughout 1949:I-2018:II, one with a period of about six years and the other with a period of about 10 years, while cycles with about 16 years and a bit less than four years also have relevant power. The wavelet power spectrum indicates that cycles with period $6 \sim 8$ years (in which the six-year cycle is particularly strong) were very intense between 1965 and 1984, while cycles of period $8 \sim 12$ years (with a particularly strong 10-year cycle) became consistently prevalent since 1984, culminating a gradual reduction of the length of a long cycle that started at periods around 14 years in the 1960s. From the early 2000s to the Great Recession, cycles of period somewhat below eight years regained power and statistical significance, but not with a comparable strength or duration of the cycles observed in the 1950s and the first half of the 1970s. After about 1995, cycles with periods larger than 16 years became statistically significant as they were until about 1975. During the final part of the sample, output gap variability was concentrated in cycles with a rather long period, confirming the findings of Crowley and Hughes Hallett(2015 and 2018) as well as Pancrazi's (2015) observation that cycles larger than the standard business cycle definition (i.e., with period larger than eight years) are increasingly relevant for macro and monetary analyses.

The time series chart of the unemployment gap broadly suggests that, as expected, it mirrors the path of the output gap, with a slight lag in most turning points. One apparent difference is that the trough of the output gap in the early 1980s recession was deeper that the trough in the Great Recession, while the unemployment rate reached a higher level in 2010 than in 1983. 
The wavelet global power spectrum identifies two dominant cycles in the unemployment rate throughout 1949:I-2018:II, the major one with a period of about 10 years and a the other with a period of 16 years, while cycles with about six years period also have notable power; it suggests, therefore, that there is a larger importance of longer cycles in the unemployment rate gap than in the output gap, when the whole sample is considered. The wavelet power spectrum seemingly confirms such suggestion, as it features extensive areas depicted in red, orange and yellow, with statistically significant power, at cycles around 16 years (comprising cycles with period apparently in the range $12 \sim 24$ years), which do not show a comparable power in the output gap. The wavelet power spectrum is, in turn, very similar to that of the output gap as regards very short cycles in the 1950s, as well as in showing that cycles with period $6 \sim 8$ years (in which the six-year cycle is particularly strong) were very strong between 1970 and 1984, and that cycles of period $8 \sim 12$ years (with a particularly strong 10-year cycle) became prevalent since 1984. Consistently with the abnormally high peak in unemployment in 2010, in the 2000s the wavelet power spectrum shows a stronger power in cycles of $6 \sim 8$ years than in the case of the output gap, but also a strong peak of variance in the second half of the 2000s in a cycle with a period slightly above the standard business cycles (the 10-year cycle). During the final part of the sample, the variance of the unemployment gap was very strong at cycles with a rather long period. Overall, the timefrequency behavior of the unemployment rate gap clearly confirms that there is the need to include in Okun's Law analyses cycles longer than the standard business cycles. 


\section{Results: Okun's Law in the Time-Frequency Domain}

We now assess the U.S. Okun's Law in the time-frequency domain, using continuous wavelet tools - the wavelet coherency, the wavelet phase-difference, and the wavelet gain between the unemployment gap

and the output gap. ${ }^{7}$ The interpretation of our econometric results proceeds along the standard approach for the coherency and phase-differences (see, e.g., Aguiar-Conraria, Martins, and Soares, 2012) - which tell us, respectively, the strength and significance of the correlation between the two series at each frequency for each moment of time, and the sign of the relation as well as the size of the lead/lag between the series; in this paper this analysis is enriched with graphs displaying the phase-difference converted into months of lead/lag. The gain is interpreted, following Aguiar-Conraria, Martins and Soares (2018), as the estimate of the absolute value of the Okun's coefficient. The phase-difference complements the information given by the gain, indicating whether the relation is positive (if the variables are in phase) or negative (if the variables are out of phase) and the timing of the relation; the graphs with the lead (lag) of output in months over time for each frequency describe more intuitively the timing of Okun's Law prevalent at each time and frequency.

To facilitate the presentation and the comparison with the literature, we provide phasedifference and gain diagrams displaying mean values corresponding to three frequency intervals: cycles of period $2 \sim 8$ years (standard business cycles), cycles of period $8 \sim 16$ years (medium-run cycles) and cycles of period $16 \sim 24$ years (long run cycles). Most literature so far has focused on the standard business cycles, but given the lenghtening of cycles documented by Crowley and Hughes Hallett(2015 and 2018) and the relevance of cycles substantially longer than standard business cycles noted by e.g., Pancrazi (2015), the inclusion of the medium and the long-run frequency bands is one important contribution of this paper to the Okun's Law literature. To facilitate the analysis of one possible nonlinearity in Okun's Law - the increase in its coefficient in recessions found in some literature - we include bars with the recession periods in the charts of the gains

\footnotetext{
${ }^{7}$ In what follows, since we always deal with wavelet-based measures, for simplicity we will avoid using the word wavelet and simply write coherency for wavelet coherency, phase-difference for wavelet phase-difference, and gain for wavelet gain.
} 
and of the output lead.

Figure 2 summarizes our results for the gaps specification of Okun's Law for the U.S. 1949:I2018:II. First, we highlight the main overall conclusions, and then go into more detail discussing the results for each frequency band.
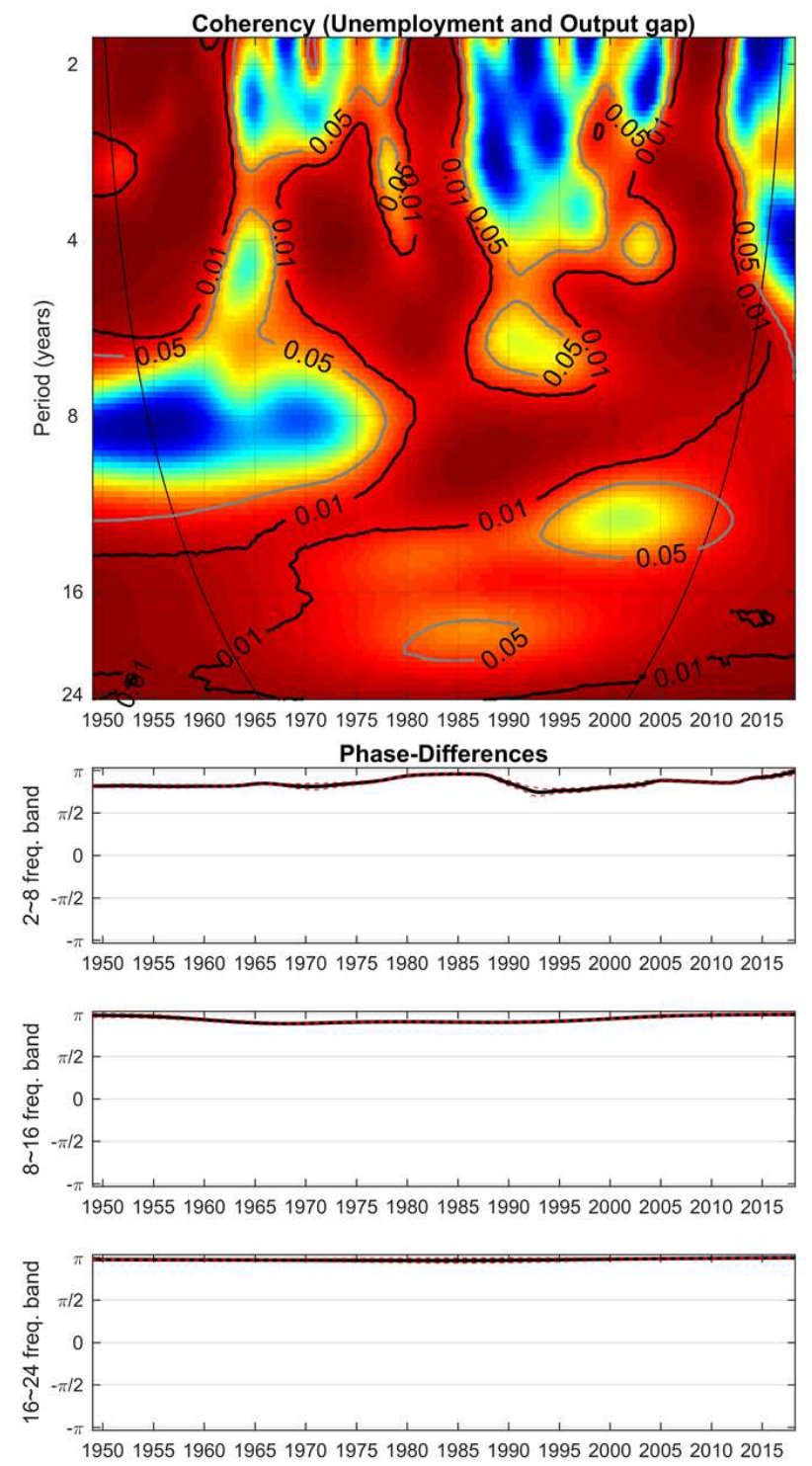
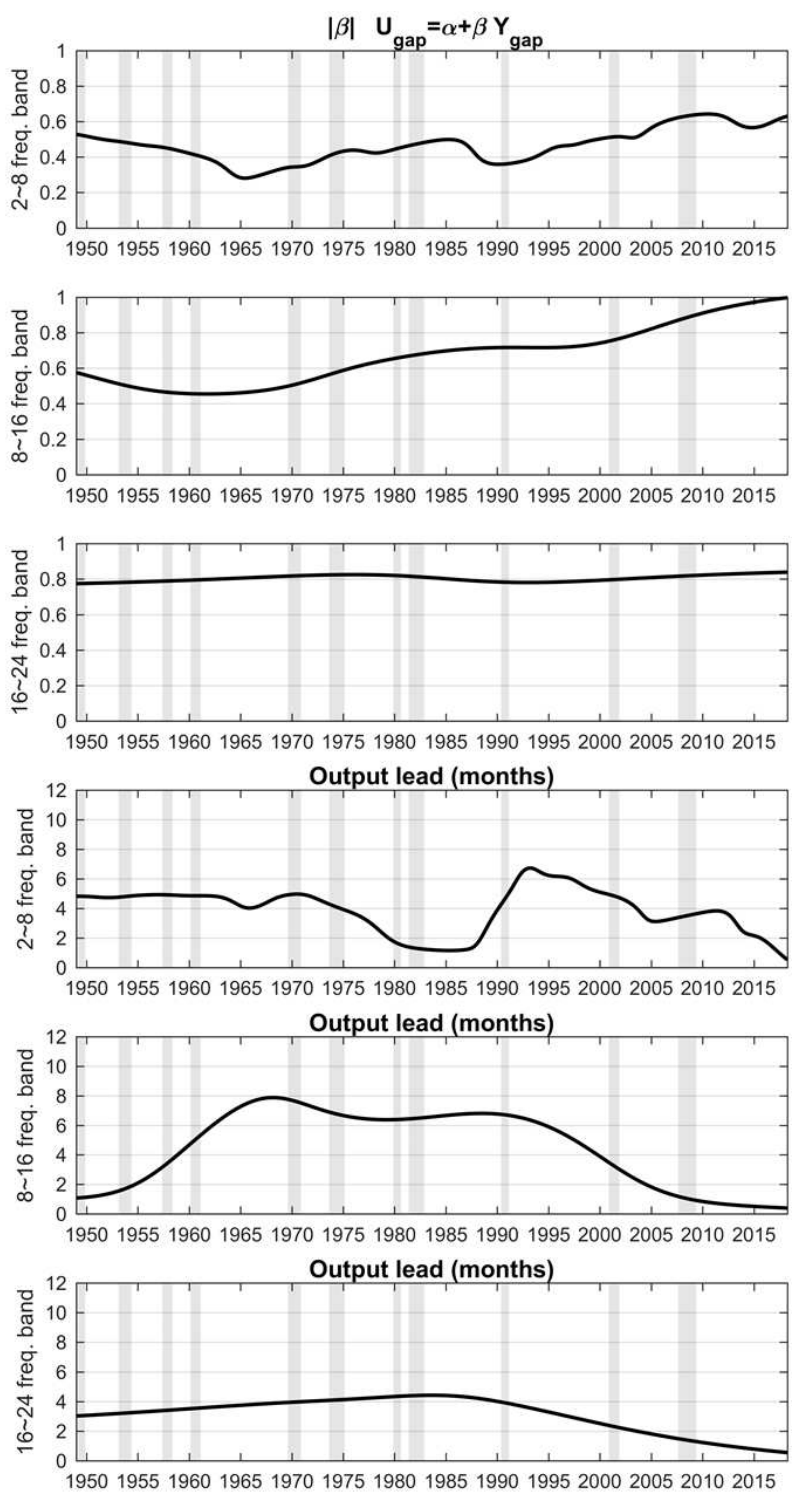

Figure 2. Left side top: Coherency between the unemployment gap and the output gap. The color code for coherency ranges from blue (low coherency - close to zero) to red (high coherency - close to one). The black (grey) contours indicate the 1\% (5\%) significance level for the wavelet coherency. The cone of influence, indicating the region affected by edge effects, is shown with a parabola-like black line.

Left side bottom: Phase-differences between cyclical fluctuations of the unemployment gap and the output gap, for the three frequency bands. Right side top: Gains from the output gap to the unemployment gap, for the three frequency bands. Right side bottom: Time-domain equivalent of the phase-differences, indicating the lead of the output gap to the unemployment gap, for the three frequency bands. 
The coherency is red and significant in most of the time-frequency space (1949:I-2018:II and two to 24 years cycles). This, on the one hand, demonstrates the prevalence and strength of the relation between cyclical unemployment and output, and, on the other hand, confirms that Okun's Law is relevant at cycles beyond the standard business cycle frequencies. Variations in coherency show that there are frequency variations in Okun's Law - which have been neglected in the literature thus far.

Phase-differences are consistently located in the interval $(\pi / 2, \pi)$, confirming that the cycles of unemployment and output are out of phase, i.e., their relation is negative. It also shows that there is some lag between changes in the output gap and changes in the unemployment gap. One can see that the lag has changed over time and differently across frequencies. Also note that this happens both for typical business cycles - period $2 \sim 8$ years - and for medium run cycles - period $8 \sim 16$ years - , which has also been neglected in the literature thus far.

Finally, the gains provide estimates of Okun's Law coefficient that are on average consistent with the rule of thumb estimate of -0.5 at the business cycle frequencies, as well as at mediumrun cycles, but in this case only until the first years of the Great Moderation. Their evolution over time is not similar across the three frequency bands - which implies that the literature that has looked extensively at asymmetries or structural breaks in Okun's coefficient is likely to have missed a relevant part of the pattern of time variation. We find no evidence of sensitivity of the gain to recessions, even at the standard business cycles frequency band, and find that the gain trends up, suggesting stronger Okun's relations, for both the business and medium run cycles since the mid-1960s, with an intensified strength after 1995 - which differs from most estimates of structural breaks in the literature of U.S. Okun's Law. The rise in Okun's coefficient after 1995 can be explained by factors such as the increase in labor market flexibility caused by institutional changes and by an increase in the share of younger workers and temporary work - see, e.g., Dixon, Lim and VanOurs (2017) -, by demographic and schooling changes - see, e.g., Ball, Leigh and Loungani (2017) - as well as by the (mostly female driven) declining trend fall in labor participation rate - see, e.g., Daly, Pedtke, Petrosky-Nadeau, and Schweinert (2018); the rise between 1965 and 1985 is a puzzle uncovered by our results. 


\subsection{Business cycles: $2 \sim 8$ years}

At the standard business cycles - period $2 \sim 8$ years - , the coherency is particularly strong and extensively significant in the following episodes: until the early 1960s - especially at cycles of period $2 \sim 6$ years -; during the oil shocks - especially at cycles with period $3 \sim 6$ years and a dominant four-year cycle -; during the early 1980s disinflation - especially at cycles of period of two years and of $4 \sim 8$ years; during the post-2003 boom and the Great Recession - in this case with special incidence in three parallel cycles, one with a two-year period, another with a $3 \sim 4$ year period, and another with a $6 \sim 8$ period. In contrast, it is noteworthy the lack of power of Okun's Law in the mid-1960s and in the first decade of the Great Moderation. In these episodes the gain indicates that the magnitude of Okun's Law coefficient has fallen, to a global minimum in 1965 of slightly less than 0.3 , and to a local minimum of slightly less than 0.4 around $1990 \mathrm{~s}^{8}$ The gain decreases consistently from the beginning of the sample - when its estimate is between 0.5 and 0.6 - until 1965 - when its estimate is slightly below 0.3 - which suggests an average estimate for that period not very different from Okun's (1962) original estimate.

From 1965 to the end of the sample the gain trends up - with the already mentioned fall in the second half of the 1980s and early 1990s, as well as another less relevant fall around 20122015 - and is estimated at somewhat above 0.6 since 2005. Such path seems consistent with the overall estimate of 0.51 of Ball, Leigh and Loungani (2017), as well as with their estimates of 0.48 until 1984:I and 0.61 since 1984:II; however, our analysis shows that at business cycles - the ones captured by the Hodrick-Prescott filtered gaps used by Ball, Leigh and Loungani (2017) - Okun's coefficient has been increasing much before the beginning of the Great Moderation; likewise, our results are not consistent with most of the literature, as, for example, the finding of a stable coefficient until the early 1980s with a sharp increase thereafter, by Grant (2018), and the finding of a small increase from 1980 to 2000 and then a substantial increase until 2010, found by Berger, Everaert and Vierke (2016).

Comparison of the gain with the recession bars strongly suggests no substantial change in the path of Okun's coefficient during recessions; while it is apparent that during the strong recessions

\footnotetext{
${ }^{8}$ In what follows, and for simplicity, we will refer to a fall (increase) in Okun's coefficient to describe a weakening (strenghtening) of the relation between unemployment and output.
} 
associated with the oil shocks, with the disinflation and with the 2007 financial crisis, the gain is rising, such rise seems part of a trend starting in 1965 and not especially associated with those episodes; therefore, our empirical framework leads to results very different from a large literature referred to in Section 2 that includes, for example, Lee (2000), Cuaresma (2003), Holmes and Silverstone (2006), Knotek II (2007), Valadkhani and Smyth (2015), Berger, Everaert and Vierke (2016), and Grant (2018); they are closer to those of Ball, Leigh and Loungani (2017), who found that the coefficient was not statistically or economically different during recessions, and consistent with the estimate of a stable coefficient for increases and decreases of the unemployment rate obtained by Fernald, Hall, Stock and Watson (2017).

Finally, our results indicate that until 1970 the unemployment gap lagged the output gap by about five months, but when the Okun relation strengthened, in the 1970s and early 1980s, the lag trended down to about one to two months, in 1980-1987; then, when the Okun's relation became somewhat looser, the lag increased to more than six months in the mid-1990s; subsequently, the lag trended down again, with a period of stable lag around four months in the financial boom and the Great Recession, to less than two months at the end of the sample; therefore, regressions that include up to two quarters of lagged output, such as Ball, Leigh and Loungani (2017), capture the maximum lag between the unemployment gap and the output gap that we estimate, but fail to capture the changes in lags over time. A fortiori, our results cast doubt on analyses that use the gaps version of Okun's Law assuming purely static quarterly regressions, as for example Kamber, Morley and Wong (2018).

\subsection{Medium run cycles: $8 \sim 16$ years}

At the medium run cycles $-8 \sim 16$ years period - , there are always some frequencies with high coherency throughout the sample period; these high coherency regions are, however, more limited until the start of the disinflation (late 1970s), while, in contrast, are especially widespread and strong between 1980 and 2000 - with dominant cycles of period $8 \sim 12$ years. In this latter episode, it is apparent a transfer of coherency from the standard business cycles - period $2 \sim 8$ years - to cycles with a more medium cycle nature $-8 \sim 12$ year period. This observation for 
medium run cycles in 1980-2000, together with the result that the Okun's Law became stronger at business cycles closer to the border of the $2 \sim 8$ years period in the 1980s and in the 2000s, implies that the relation between unemployment and output gaps has, to some extent, progressively encompassed longer cycles throughout the last decades (before the Great Recession). This result brings together the results of Crowley and Hughes Hallett(2015 and 2018) - who document that longer cycles became more important to explain the variance of output - and our similar results for unemployment (see Figure 2).

The pattern of evolution of the gain over time for this frequency band is similar to that of the gain for standard business cycles, although smoother and shifted up to stronger coefficients; starting from an estimate of 0.6, the gain falls until 1965, when it reaches a value slightly above 0.4, and then trends up until the end of the sample with only some interruption of that trend in 1985-1995; since 1980 the gain is above 0.6 and since 2005 our estimate of the gain is above 0.8 ; therefore, our estimates of the Okun's Law coefficient for medium run cycles suggest a stronger relation between the unemployment gap and the output gap than at the standard business cycles - a result that many applied analyses do not capture, as they focus only on business cycles. Considering the results for both $2 \sim 8$ and $8 \sim 16$ year cycles, our overall average estimate is consistent with that of about 0.7 obtained by Kamber, Morley and Wong (2018) for 1948:I-2016:II, suggesting that their Beveridge-Nelson filter captures frequencies above the standard business cycle threshold of eight years.

Comparison of the gain for $8 \sim 16$ years cycles with the recession bars suggests that there is no substantial change in Okun's coefficient during recessions. The lag of the cyclical fluctuations of the unemployment gap relative to those of the output gap at these frequencies increase since the beginning of the sample until it reaches eight months (almost three quarters) in the late 1960s; it then decreases to six quarters at 1980 and upholds such lag during the 1980s, after which the lag starts decreasing to less than two months by the end of the period; it is noteworthy that the lags change more markedly than at standard business cycles, and that the most standard dynamic regressions in the literature - including two quarters of lags - would not capture the whole dynamics of Okun's Law for cycles of $8 \sim 16$ years between the early 1960s and 1995, let alone their sizable variation over time. 


\subsection{Long run cycles: $16 \sim 24$ years}

At long run cycles - period $16 \sim 24$ years -, coherency is strong throughout, although more limited in 1980-1995 - when it is not significant at 5\% for some frequencies - and shows no episodes of specially strong coherency. The gain is consistently stable at 0.8 , an estimate considerably above the standard estimates in the literature, suggesting that, by disregarding longer fluctuations, the literature is not capturing important dimensions of Okun's Law. The phase-differences imply that the unemployment gap lags the output gap by about three months at the start of the sample, and that the lag smoothly increases to four months until 1985 - the period of more limited coherency; then, the lag decreases to reach less than two months by the end of the sample period. 


\section{Conclusions}

In this paper, we argue that recent literature on Okun's Law that has focused on changes, over time, in the relationship between cyclical unemployment and cyclical output lacks the analysis of possible changes in its lags over time as well as of changes in the relation across cyclical frequencies. Using a set of continuous wavelet tools, we estimate the U.S. Okun's Law coefficient during 1949:I2018:II, simultaneously allowing for variation over time as well as variation in the timing of the relation and variation across frequencies. We uncover new stylized facts about the U.S. Okun's Law in the past seven decades that would be hard to detect either in the pure time-domain or in the pure frequency-domain. Using our preferred specification, the gaps version, we found the Okun's relation to being strong and statistically significant for most of the time at all cyclical frequencies. The first differences specification (in the appendix) delivers results very similar at the business cycle frequencies; at lower frequencies, the estimated Okun relation is weaker.

We found the Okun relation to be weak at business cycles in the first ten years of the Great Moderation, a period during which medium-run cycles are particularly strong. Okun's relation becomes relevant again at business cycles in the post-2003 boom, the Great Recession, and the early period of the subsequent recovery. We observe a gradual strengthening of Okun's Law at increasingly longer cycles, which is in line with the literature that has detected and explained a transfer of volatility from shorter to longer cycles - Crowley and Hughes Hallett (2015 and 2018).

Our estimates of the Okun's Law coefficient exhibit substantial variation over time, with different patterns in different frequency bands. However, we find no evidence of any sensitivity of Okun's coefficient to recessions. At the business cycles frequency band, the average of our estimates for 1949-2018 is consistent with the usual rules of thumb as well as with estimates obtained by authors that have assessed the U.S. Okun's Law with comparable measures of cyclical unemployment and output - e.g., Ball, Leigh and Loungani (2017). Over time, the coefficient is not stable: after decreasing until 1965 to an estimate of about 0.3, the Okun's coefficient for $2 \sim 8$ years cycles starts trending up, ending with an estimate above 0.6 in 2010. Our estimates of Okun's coefficient at the medium run cycles also point to a strengthening of the relationship between cyclical unemployment and cyclical output since 1965. In contrast, our estimate of Okun's 
coefficient in the long run cycles is quite stable at around 0.8 during the whole period. Overall, our results suggest that disregarding cycles of lower frequency than business cycles underestimates the relation between the unemployment and the output gaps.

Our approach uncovers a substantial variation of the lead of output over unemployment. At the business cycle frequencies, the lead falls from five months in 1970 to about two months in 1980, then increases between 1987 and 1993 (when the Okun's relation was weaker) to more than six months, and subsequently trends down again to about four months in the financial boom and the Great Recession. At medium-run cycles, the lead of output oscillates between eight and six months between the late 1960s and the early 1990s, which is somewhat longer than most regressions in the literature, and then gradually falls to less than two months after the Great Recession. At long run cycles, the lead gradually increases to four months in 1985 and then systematically falls to less than two months. Therefore, regressions that include up to two quarters of lagged output, such as Ball, Leigh and Loungani (2017), almost capture the lead of output over unemployment that we estimate but fail to capture its changes over time. Our results endorse more flexible time-series regressions such as Fernald, Hall, Stock and Watson (2017), who estimate the first differences version of Okun's Law with two lags and two leads.

Finally, our estimates of Okun's coefficient and the lead of output, at business cycles frequencies complement Galí, Smets and Wouters (2012) view that the recoveries after the troughs of 1991:I, 2001:IV and 2009:II were all similarly slow, as we find that, after 1991:I, Okun's coefficient was lower and the lead of output higher than after 2001:IV, and that, following the 2009:II trough, the coefficient was at its highest estimate throughout 1949-2018, and the lead of output was much lower than in the 1990s. Such strengthening of Okun's Law following the Great Recession squares well with the findings of Fernald, Hall, Stock and Watson (2017) that it was the output that fell short of the typical recovery in the seven years after 2009:II, not unemployment.

\section{Acknowledgments}

Research at NIPE was carried out within the funding with COMPETE reference number POCI01-0145-FEDER-006683 (UID/ECO/03182/2013), with the FCT/MEC's (Fundação para a Ciência e a Tecnologia, I.P.) financial support through national funding and by the ERDF through the Operational Programme on "Competitiveness and Internationalization - COMPETE 2020 under the PT2020 Partnership Agreement. 
Research at Cef.up has been financed by Portuguese Public Funds through FCT (Fundação para a Ciência e a Tecnologia) and by the European Regional Development Fund through COMPETE 2020 - Programa Operacional Competitividade e Internacionalização - in the framework of the project UID/ECO/04105/2013. 


\section{References}

[1] Aguiar-Conraria, Luís, Manuel M. F. Martins, and Maria J. Soares (2012) "The Yield Curve and the Macro-Economy Across Time and Frequencies" Journal of Economic Dynamics and Control, 36, 1950-1970.

[2] Aguiar-Conraria, Luís, Manuel M. F. Martins, and Maria J. Soares (2018) "Estimating the Taylor Rule in the time-frequency domain" Journal of Macroeconomics, 57, 122-137.

[3] Aguiar-Conraria, Luís, and Maria J. Soares (2014) "The Continuous Wavelet Transform: Moving beyond Uni-and Bivariate Analysis" Journal of Economic Surveys, 28, 344-375.

[4] Aguiar-Conraria, Luís, Maria J. Soares and Rita Sousa (2018) "California's carbon market and energy prices: a wavelet analysis" Philosophical Transactions of the Royal Society A, 376, 20170256.

[5] Arai, Natsuki (2016) "Evaluating the Efficiency of the FOMC's New Economic Projections." Journal of Money, Credit and Banking 48, 1019-1049.

[6] Ball, Laurence, Daniel Leigh and Prakash Loungani (2017) "Okun's Law: Fit at 50?." Journal of Money, Credit and Banking, 49, 1413-1441.

[7] Berger, Tino, Gerdie Everaert, and Hauke Vierke (2016) "Testing for time variation in an unobserved components model for the US economy." Journal of Economic Dynamics and Control 69, 179-208.

[8] Bjørnland, Hilde C., Vegard H. Larsen, and Junior Maih (2018). "Oil and Macroeconomic (In)stability." American Economic Journal: Macroeconomics, 10, 128-51.

[9] Brock, William A., Steven N. Durlauf, and Giacomo Rondina (2008) "Frequency-specific effects of stabilization policies." American Economic Review, 98, 241-45.

[10] Brock, William A., Steven N. Durlauf, and Giacomo Rondina (2013) "Design limits and dynamic policy analysis." Journal of Economic Dynamics and Control 37, 2710-2728.

[11] Canova, Fabio (2007). Methods for Applied Macroeconomic Research. Princeton and Oxford: Princeton University Press, pp. 492.

[12] Cazes, Sandrine, Sher Verick, and Fares Al Hussami (2013) "Why did unemployment respond so differently to the global financial crisis across countries? Insights from Okun's Law." IZA Journal of Labor Policy 2:10, pp.18.

[13] Coibion, Olivier, and Yuriy Gorodnichenko (2011) "Monetary Policy, Trend Inflation and the Great Moderation: An Alternative Interpretation." American Economic Review, 101, 341-370.

[14] Crowley, Patrick M. and Andrew Hughes Hallett (2015) "Great moderation or "Will o' the Wisp"? A time-frequency decomposition of GDP for the US and UK." Journal of Macroeconomics 44, 82-97.

[15] Crowley, Patrick M. and Andrew Hughes Hallett (2018) "What causes business cycles to elongate, or recessions to intensify?" Journal of Macroeconomics 57, 338-349. 
[16] Cuaresma, Jesus Crespo (2003) "Okun's law revisited." Oxford Bulletin of Economics and Statistics 65, 439-451.

[17] Daly, Mary C., John G. Fernald, Òscar Jordà, and Fernanda Nechio (2017) "Shocks and Adjustments." Federal Reserve Bank of San Francisco Working Paper 2013-32, pp. 37.

[18] Daly, Mary C., Joseph H. Pedtke, Nicolas Petrosky-Nadeau, and Annemarie Schweinert (2018) "Why Aren’t US Workers Working?" Federal Reserve Bank of San Francisco Economic Letter, 24.

[19] Dixon, Robert, Guay C. Lim, and Jan C. van Ours (2017) "Revisiting the Okun relationship." Applied Economics 49, 2749-2765.

[20] Engle, Robert (1976) "Interpreting spectral analyses in terms of time-domain models." Annals of Economics and Social Measurement, 5(1), 89-109.

[21] Fernald, John G., Robert E. Hall, James H. Stock, and Mark W. Watson (2017) "The disappointing recovery of output after 2009." National Bureau of Economic Research Working Paper No. w23543.

[22] Gallegati, Marco, Mauro Gallegati, James . B. Ramsey and Will Semmler (2011), "The US Wage Phillips Curve across Frequencies and over Time." Oxford Bulletin of Economics and Statistics, 73: 489-508.x

[23] Gallegati, Marco and Willi Semmler (2014) Wavelet Applications in Economics and Finance, Springer International Publishing (2014)

[24] Galí, Jordi, Frank Smets, and Rafael Wouters (2012) "Slow recoveries: A structural interpretation." Journal of Money, Credit and Banking 44, 9-30.

[25] Gordon, Robert J. (2010a) "Okun's law and productivity innovations." American Economic Review 100, 11-15.

[26] Gordon, Robert J. (2010b) "The demise of Okun's law and of procyclical fluctuations in conventional and unconventional measures of productivity." Presented at NBER Summer Institute, PRBB Research Meeting, Cambridge MA, July 21.

[27] Grant, Angelia L. (2018) "The Great Recession and Okun's law." Economic Modelling 69, 291-300.

[28] Hodrick, Robert J. and Edward C. Prescott (1997) "Postwar U.S. Business Cycles: An Empirical Investigation." Journal of Money, Credit and Banking, 29, 1-16.

[29] Holmes, Mark J., and Brian Silverstone (2006) "Okun's law, asymmetries and jobless recoveries in the United States: A Markov-switching approach." Economics Letters 92, 293-299.

[30] Kamber, Güneş, James Morley, and Benjamin Wong (2018) "Intuitive and reliable estimates of the output gap from a Beveridge-Nelson filter." Review of Economics and Statistics, 100, $550-566$

[31] Knotek II, Edward S. (2007) "How useful is Okun's law?." Economic Review-Federal Reserve Bank of Kansas City 92, 73-103. 
[32] Lee, Jim (2000) "The robustness of Okun's law: Evidence from OECD countries." Journal of Macroeconomics 22, 331-356.

[33] Mandler, Martin, and Michael Scharnagl (2014) "Money growth and consumer price inflation in the euro area: a wavelet analysis." Discussion Paper, Deutsche Bundesbank No 33/2014.

[34] Meyer, Brent, and Murat Tasci (2012) "An unstable Okun's Law, not the best rule of thumb." Federal Reserve Bank of Cleveland Economic Commentary 2012-08.

[35] Okun, Arthur. M. (1962) "Potential GNP: Its Measurement and Significance". Proceedings of the Business and Economic Statistics Section of the American Statistical Association 7, 98-104.

[36] Owyang, Michael T., and Tatevik Sekhposyan (2012) "Okun's law over the business cycle: was the great recession all that different?." Federal Reserve Bank of St. Louis Review 94 September/October 399-418.

[37] Pancrazi, Roberto (2015) "The Heterogenous Great Moderation." European Economic Review, 74, February, 207-228.

[38] Prachowny, Martin FJ. (1993) "Okun's law: theoretical foundations and revised estimates." The Review of Economics and Statistics, 331-336.

[39] Silvapulle, Paramsothy, Imad A. Moosa, and Mervyn J. Silvapulle (2004) "Asymmetry in Okun's law." Canadian Journal of Economics 37, 353-374.

[40] Valadkhani, Abbas, and Russell Smyth (2015) "Switching and asymmetric behaviour of the Okun coefficient in the US: Evidence for the 1948-2015 period." Economic Modelling 50, $281-290$.

[41] Verona, Fabio (2020) "Q, Investment, and the Financial Cycle". Oxford Bulletin of Economics and Statistics doi:10.1111/obes.12321. 


\section{Appendix - Unemployment change and output growth}

In Figure A.1 we present a description of the data to be used in the first differences specification of Okun's Law. Output growth is the change in the natural logarithm of real output from the previous quarter, and unemployment change is the difference between the unemployment rate of each quarter and the previous one, both measured in percentage points. As in Figure 1, in addition to the standard time-series graph (top pictures), we present the wavelet-based global power spectra (bottom pictures) and the wavelet power spectra (pictures in the middle).

Figure A.1 confirms the well-known result - see, e.g., Canova (2007) - that the first difference operator is a filter that strongly mitigates fluctuations at medium-to-long run periods and exacerbates fluctuations at short cycles: the wavelet global power spectra of output growth and unemployment rate changes - which are very similar, although indicating a higher overall variance of unemployment changes - are both dominated by a cycle with a two-year period; these are followed by a cycle with a four-year period and another with a six-year period, which have a smaller but still relevant power; cycles with periods of about 10 and 16 years, which are very important in the unemployment and output gaps, feature a small variance throughout 1949:I-2018:II.

In spite of the inevitable noise, the time-series charts show the Great Moderation between 1984 and 2007, as well as the great instability of both series in the 1950s, early 1960s, and in the 1970s. The Great Recession also stands out very clearly, and then the charts suggest that after 2010 both series resumed a behavior similar to that of the Great Moderation. The time series charts also indicate very clearly that at the major turning points, unemployment changes are a mirror of output growth, moreover suggesting a much more contemporaneous relation than in the case of the gaps. 

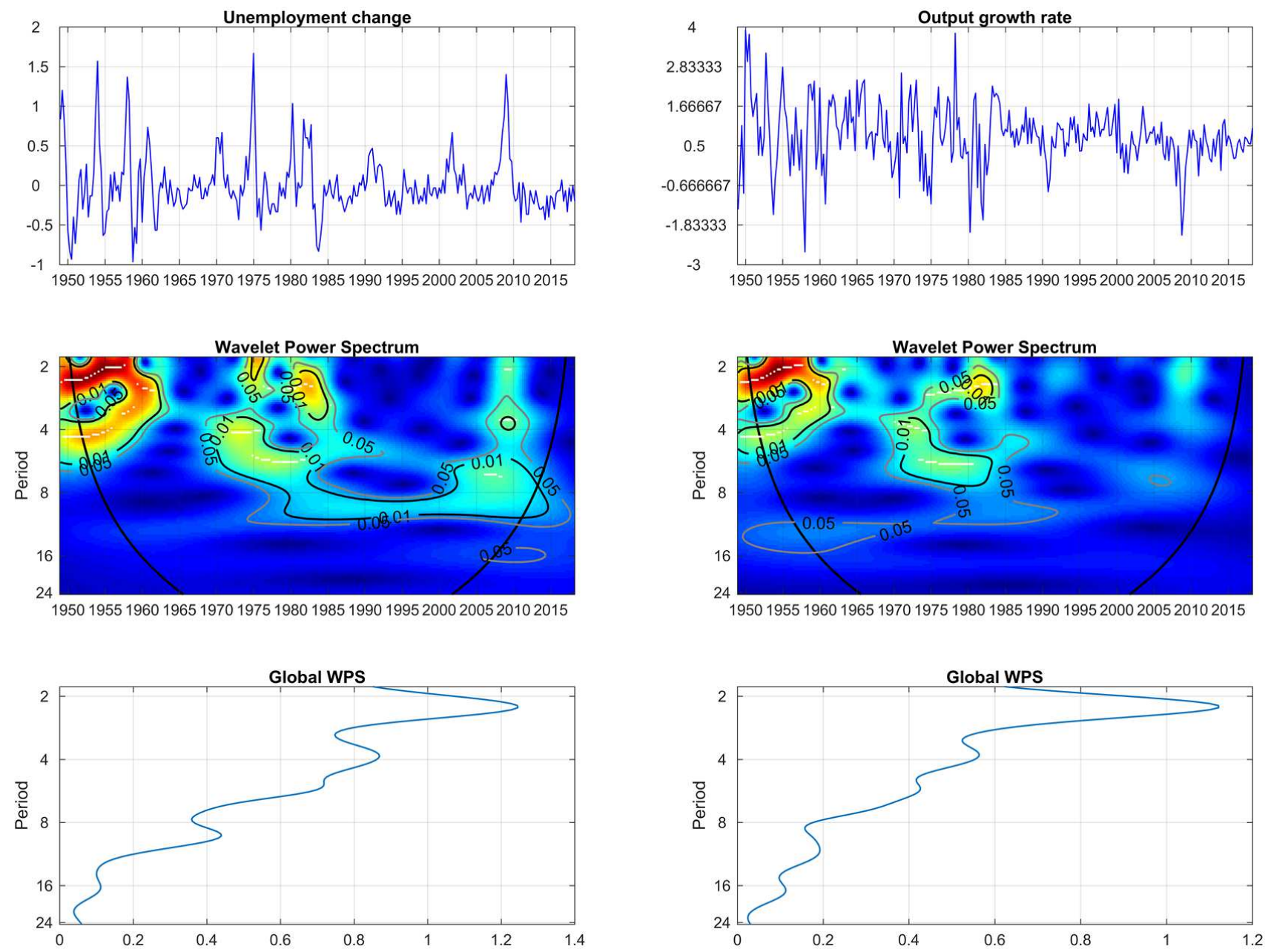

Figure A.1. Top: Time-series plots of the quarterly growth rate of real output and the quarterly change in the unemployment rate. Bottom: Global power spectrum, showing the variance of each series at each frequency in the overall sample. Middle: Wavelet power spectrum, showing the variance of each series at each time-frequency locus. The color code for power ranges from blue (low power) to red (high

power). The white lines show the local maxima of the wavelet power spectrum. The black (grey) contours indicate the 1\% (5\%) significance level. The cone of influence, which indicates the region affected by edge effects, is shown with a parabola-like black line. (for interpretation of the references to color in this legend, the reader is referred to the web version of this article.)

\subsection{Okun's Law in first differences}

Figure A.2 summarizes our results for the changes specification of Okun's Law for the U.S. 1949:I2018:II. As before, we first highlight the main overall conclusions, and then go into more detail through the results for each frequency band. 

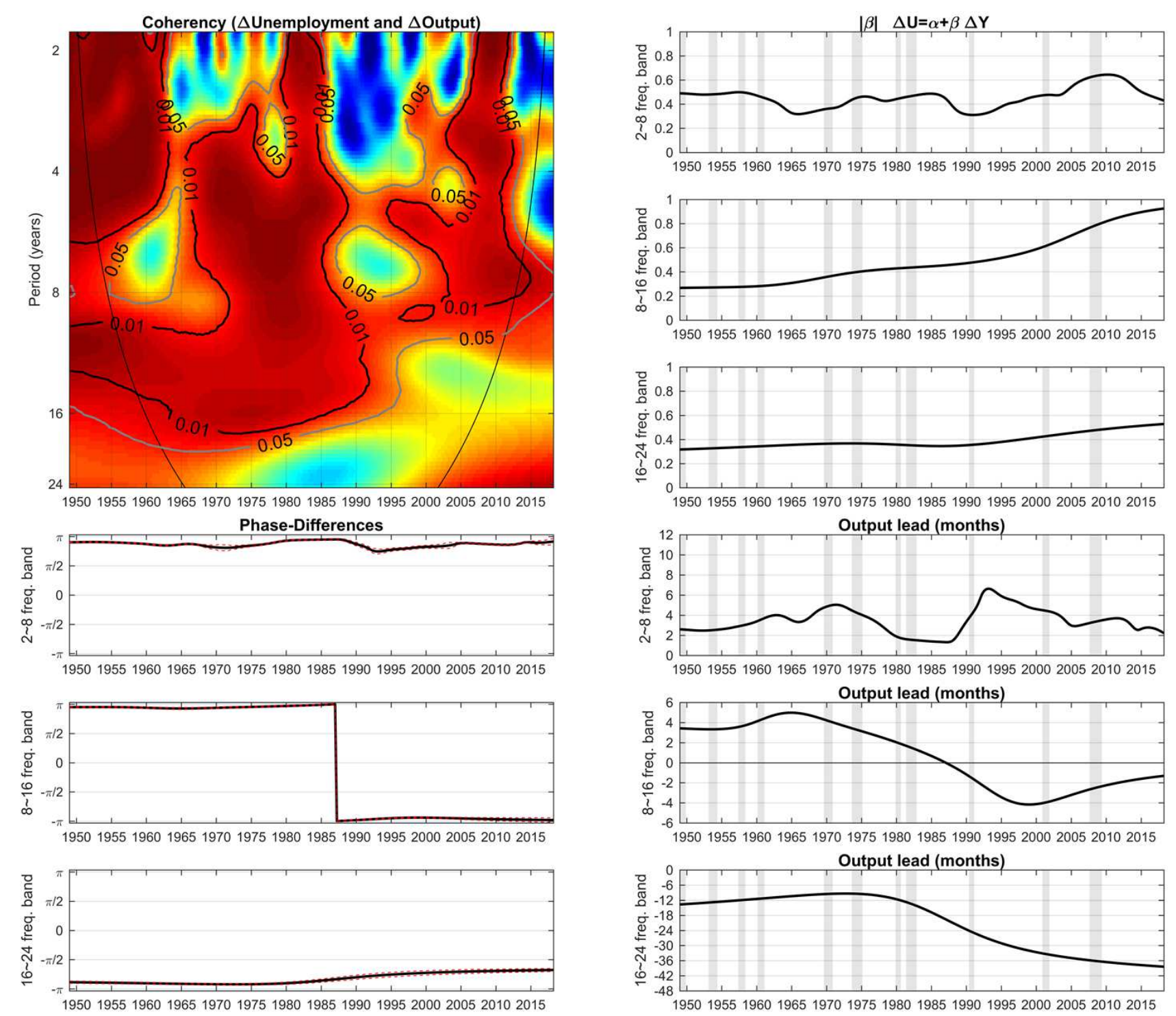

Figure A.2. Left side top: Coherency between unemployment changes and output growth. The color code for coherency ranges from blue (low coherency - close to zero) to red (high coherency - close to one). The black (grey) contours indicate the 1\% (5\%) significance level for coherency. The cone of influence, indicating the region affected by edge effects, is shown with a parabola-like black line. Left side bottom: Phase-differences between cyclical fluctuations of unemployment changes and output growth, for the three frequency bands. Right side top: Gains from output growth to unemployment changes, for the three frequency bands. Right side bottom: Time-domain equivalent of the phase-differences, indicating the lead of output gowth to unemployment changes, for the three frequency bands.

The coherency between changes is remarkably similar to the one between the gaps at the standard business cycles - period $2 \sim 8$ years - , but less so at the medium run cycles - period $8 \sim 16$ years - and is very different (and broadly non significant) at the longer cycles - period $16 \sim 24$ years. As expected, the changes specification is a useful robustness check of Okun's 
Law, but more limited, by construction, to shorter cycles; the variation in the coherency across frequencies and over time confirms the worth of our econometric approach.

The phase-differences are consistently located in the interval $(\pi / 2, \pi)$ for business cycle frequencies and when the medium run cycles feature a significant coherency in widespread regions (with a small puzzling exception to be addressed below), thus confirming that the relation is negative and that changes in unemployment typically lag changes in output; the lag has changed over time and across frequencies, again confirming the usefulness of our econometric approach.

The gains provide estimates of Okun's Law coefficient that are on average consistent with estimates in recent literature - e.g., -0.43 in Ball, Leigh and Loungani (2017) - at the business cycle frequencies as well as at medium-run cycles (but, in this case, only until the first years of the Great Moderation). Their variation over time is not similar across the two relevant frequency bands, implying that our approach adds value to the literature that has looked at asymmetries or structural breaks in Okun's coefficient without considering frequency-domain patterns. Finally, we find no evidence of sensitivity of the gain to recessions, even at the standard business cycles frequency band, and find that the gain trends up, implying a gradually stronger Okun relation for both the business and medium run cycles since the mid-1960s, with an intensified strength after 1995; these results confirm those obtained with the gap specification, thus attesting their robustness; and they differ from most estimates of structural breaks in the literature of U.S. Okun's Law; as mentioned above, it is easier to explain the rise in Okun's coefficient after 1995 than the rise that we uncover between 1965 and 1985.

\section{Business cycles: $2 \sim 8$ years}

At standard business cycles frequencies, the coherency is remarkably similar to the coherency of the gaps specification: it is particularly strong and extensively significant until the mid-1960s especially at cycles of period $2 \sim 6$ years; during the oil shocks - especially at cycles with period $4 \sim 8$ years; during the early 1980s disinflation - especially at cycles of period of two years and of $4 \sim 8$ years; during the post-2003 boom and the Great Recession - in the latter especially in three parallel cycles, one with a two-year period, another with a four-year period, and another with a period close to eight years. It is also noteworthy, as in the gaps version, the lack of power 
of Okun's Law in the mid-1960s and in 1985-1995.

The evolution of the gain is again similar to that of the gaps specification, with only some levels shifts: after falling from 0.5 since late 1950s, from 1965 onwards (when its estimate is about 0.3), the Okun's coefficient trends up until 2010, reaching a value slightly above 0.6. Such path is consistent with the overall estimate of -0.43 of Ball, Leigh and Loungani (2017), as well as with their estimates of -0.42 until 1984:I and -0.51 since 1984:II. However, the gain of the first differences specification confirms that, as in the gaps specification, at cycles of period $2 \sim 8$ years - the ones captured by Ball, Leigh and Loungani (2017) - Okun's coefficient has been increasing much before the beginning of the Great Moderation. Likewise, our results improve on the literature that found structural breaks - either in the mid-1980s, 1990 or 2000 - by showing that the coefficient was not stable within each sub-samples. Also in line with the findings obtained in the gaps specification, comparison of the gain with the recession bars strongly suggests no substantial change in the path of Okun's coefficient during recessions.

Therefore, our empirical framework leads to results very different from a large literature referred to in Section 2. They are compatible with those of Ball, Leigh and Loungani (2017), who found that the coefficient was not statistically or economically different during recessions.

Finally, the phase-differences imply a pattern of output lead that is identical to the one estimated for the gaps specification, with the only exception that, until 1970, the lead is relatively stable at around five months (with a transitory fall to four months in the mid-1960s) rather than starting from below three months; therefore, most dynamic regressions in the literature - that include up to two quarters of lagged output, such as Ball, Leigh and Loungani (2017) - capture the maximum lag between unemployment changes and output changes that we estimate, but fail to capture substantial changes in the pattern of lags over time. A fortiori, our results cast doubt on analyses that use the first differences version of Okun's Law assuming purely static quarterly regressions, as for example Arai (2016).

\section{Medium run cycles: $8 \sim 16$ years}

At the medium run cycles - period $8 \sim 16$ years - the coherency is strong and very significant throughout the whole range of frequencies until 1995, but not thereafter - except for a small 
frequency range close to the border of eight years cycles that is associated with a very high coherency inside the traditional business cycles frequencies. Coherency is particularly strong in the 1950s and first half of the 1960s, for a cycle with dominant period of $10 \sim 12$ years, between the late 1960s and the early 1980s, for a dominant cycle with period around 16 years, and in 1975-1985, for cycles with period of $8 \sim 12$ years.

Differently from what we found in the gaps specification, in the first differences specification it is not apparent any transfer of coherency from the standard business cycles to medium run cycles - if anything, this specification captures a shift of coherency, encompassing longer cycles, within the business cycle frequencies - cycles with period $2 \sim 8$ years.

The gain is stable at 0.3 until 1965, and then gradually increases to 0.5 in 1995; subsequently, it keeps on rising, to about 0.9 by 2015, but the estimates after 1995 can hardly be considered statistically significant. Comparison of the gain for $8 \sim 16$ years cycles with the recession bars show no change in Okun's coefficient during recessions - a result similar to the one found in the gaps specification. The lag of the cyclical fluctuations of unemployment relative to output at these frequencies is quite stable at four months until 1960 and rises to five months in 1965; thereafter, the lag gradually decreases and turns into a lead of unemployment by 1987; the lead gradually increases and is estimated at four months in 1995, when the coherency starts indicating that the Okun relation lacks statistical significance.

Our finding of a lead of unemployment relative to output that is statistically significant during 1987-1995 and subsequently at some very specific frequencies of this band, is a somewhat unusual result. The most standard dynamic regressions in the literature - which include lags of output and not leads - would not capture the whole dynamics of Okun's Law for cycles of $8 \sim 16$ years between the late 1980s and the mid-1990s. A notable recent exception is Fernald, Hall, Stock and Watson (2017), who estimate the first differences version of Okun's Law with two lags and two leads, finding an estimate of 0.67 for 1981:III-2016:II that seems to capture more thoroughly the relation between output and unemployment changes at cyclical frequencies.

\section{Long run cycles: $16 \sim 24$ years}

At long run cycles - $16 \sim 24$ years period - the coherency is overall weak and not statistically 
significant. The lack of significance of the first differences specification of the Okun's Law is not a surprise, given the statistical properties of the first difference filter and the lack of global power of both time series at these cycles (see Figure A.1; indeed, it may be argued that even the strong and significant coherency found for $8 \sim 16$ years cycles comes somewhat as a surprise). In view of the lack of statistical significance, we refrain from analyzing the gain and the phase-differences for cycles of period $16 \sim 24$ years. 